\title{
DESARROLLO DE UN SISTEMA DE INFORMACIÓN PARA LA EMPRESA Datecsa S.A.
}

\section{THE DEVELOPMENT OF AN INFORMATION SYSTEM FOR THE COMPANY Datecsa S.A}

\begin{abstract}
The following document presents: the survey, implementation and analysis of a system to manage the information of the company Datecsa Colombiana S.A., It initially takes into account the requirements of the users of the system, based on this, the most viable technological option is selected, for its subsequent implementation. Finally, the security of the developed system is analysed which leads to demonstrate its robustness.
\end{abstract}

Key words: databases, encryption, information system, use cases, virtual private network.

\section{RESUMEN}

En el siguiente documento se presenta un estudio, implementación y análisis de un sistema para administrar la información de la empresa Colombiana Datecsa S.A. Inicialmente, se tienen en cuenta los requerimientos de los usuarios del sistema, a partir de esto se selecciona la opción tecnológica más viable, para su posterior implementación. Finalmente, se analiza la seguridad del sistema desarrollado, lo que conlleva a demostrar su robustez.

Palabras claves: bases de datos, casos de uso, encriptación, red privada virtual, sistema de información.
Nelson Javier Reyes

Ingeniero en Telecomunicaciones Ingeniero de desarrollo Banco de Bogotá

nreyes1@bancodebogota.com.co

Bogotá, Colombia

\section{Andrés Castiblanco}

Ingeniero en Telecomunicaciones Ingeniero Getronics hash.ares@gmail.com

Bogotá, Colombia

\section{Luis Fernando Pedraza}

Magister en Ciencias de la Información y las Comunicaciones

Docente planta de la Universidad Distrital Francisco José de Caldas 1fpedrazam@udistrital.edu.co Bogotá, Colombia

Tipo: Artículo reporte de caso

Fecha de Recepción: Junio 10 de 2013 Fecha de Aceptación: Agosto 6 de 2013 


\section{INTRODUCCIÓN}

Datecsa S.A es una empresa que presta servicios de soluciones documentales y audiovisuales, por tanto con el fin de mantener la calidad de sus servicios mejora continuamente sus procesos, a partir de diagnósticos realizados a través de auditorías. Lo anterior, ha demostrado la necesidad de desarrollar un sistema totalmente dinámico, seguro y conveniente para la ejecución de los planes de negocio de la compañía, que permita mejorar la prestación actual.

A continuación se presentan casos de éxito sobre el desarrollo de sistemas de información, que han servido de base para la consecución del presente proyecto.

En $[1,2]$ se presentan metodologías para el desarrollo de sistemas de información para satisfacer las necesidades de los clientes. En [3], se desarrolla un sistema de información para organizar la información y evaluar el desempeño investigativo de las universidades de Malasia. En [4] se realiza una solución para administración de operaciones en terreno usando dispositivos móviles y mensajería SMS.

\section{METODOLOGÍA DEL PROYECTO}

Se llevaron a cabo diferentes etapas para la consecución de los resultados, como son:

- Levantamiento general de la información.

- Análisis de la situación actual.

- Análisis de alternativas.

- Implementación de la solución de acuerdo al análisis previo.

- Pruebas generales.

- Capacitación de usuarios.

Lo anterior, aporta un estudio y solución al sistema de información, que minimizará problemas como retrasos, errores, gestión obsoleta e inconvenientes, y permitirá el cumplimiento a cabalidad de las normas de calidad.

\section{ESPECIFICACIONES DEL DESARROLLO}

Para el análisis de las diferentes opciones de desarrollo, se estudian los factores requeridos en la compañía y por el personal. En la figura 1 se muestran las variables porcentuales de los requerimientos para la solución.

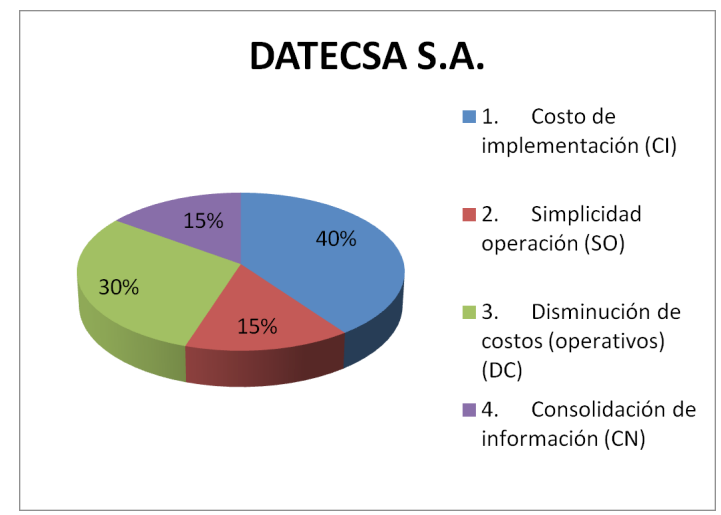

Figura 1. Niveles porcentuales requeridos para el desarrollo de la solución en la compañía

En la figura 2 se presenta los niveles porcentuales de uso que tendría la aplicación en el equipo de trabajo de la compañía.

\section{Grupo de trabajo}

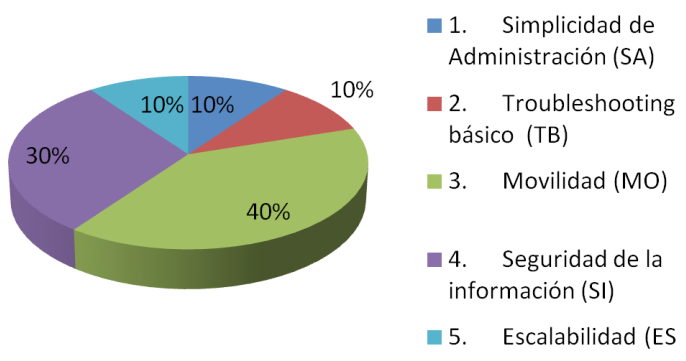

Figura 2. Niveles porcentuales de uso de la solución en el grupo de trabajo

Luego de conocer los requerimientos para el diseño, se procede a evaluar cuantitativamente las diferentes soluciones tecnológicas, con valores entre 0 y 10 (donde 0 es la calificación más baja y 10 es la calificación más alta), teniendo en cuenta las características de cada solución, tal como se muestra en la tabla 1.

\subsection{Análisis de soluciones}

En la tabla 1, aparecen las especificaciones de 
las opciones con sus posibles combinaciones, argumentado las ventajas y desventajas de cada una y con base en diferentes software [5-7], además ponderadas de acuerdo a la pertinencia entre ellas. Teniendo en cuenta los niveles porcentuales requeridos citados anteriormente en las figuras 1 y 2 , se tomó como medida de puntuación la ecuación (1).
PT: promedio de puntaje total para la solución contemplada

\section{PI: puntaje individual por ítem considerado}

CI, SO, DC, CN, SA, TB, MO, SI, ES: porcentajes de requerimientos dispuestos por la empresa y el grupo de trabajo, presentados en las figuras 1 y 2.

Donde:

$$
P T=\frac{(P I \cdot C I)+(P I \cdot S O)+(P I \cdot D C)+(P I \cdot C N)+(P I \cdot S A)+(P I \cdot T B)+(P I \cdot M O)+(P I \cdot S I)+(P I \cdot E S)}{2}
$$

Tabla 1. Evaluación de tecnologías

\begin{tabular}{|c|c|c|c|c|}
\hline Opción & $\begin{array}{l}\text { Tecnología } \\
\text { propuesta }\end{array}$ & Ventajas & Desventajas & $\begin{array}{l}\text { Puntuación } \\
\text { total } \\
1-10\end{array}$ \\
\hline \multirow{3}{*}{$\begin{array}{l}\text { Aplicación } \\
\text { independiente en } \\
\text { móvil }\end{array}$} & \multirow{3}{*}{ Java } & Fácil implementación & $\begin{array}{l}\text { Visitas continuas a un servidor } \\
\text { central para descarga de } \\
\text { información }\end{array}$ & \multirow{3}{*}{6,5} \\
\hline & & Costos de desarrollo bajos & $\begin{array}{l}\text { Seguridad de la información } \\
\text { sujeta a problemas con el móvil }\end{array}$ & \\
\hline & & $\begin{array}{l}\text { Eliminación de orden impresa } \\
\text { en papel }\end{array}$ & Sin integración con el cliente & \\
\hline \multirow{4}{*}{$\begin{array}{l}\text { Aplicación residente } \\
\text { en servidor }\end{array}$} & .NET & Costos de desarrollo bajos & Difícil implementación & \multirow{4}{*}{6} \\
\hline & $\begin{array}{l}\text { Visual } \\
\text { Basic }\end{array}$ & $\begin{array}{l}\text { Eliminación de orden impresa } \\
\text { en papel }\end{array}$ & $\begin{array}{l}\text { Necesidad de instalación de } \\
\text { métodos adicionales y/o reglas } \\
\text { en firewall }\end{array}$ & \\
\hline & \multirow{2}{*}{ Java } & \multirow{2}{*}{$\begin{array}{l}\text { Capacidad de verificación de } \\
\text { datos en línea }\end{array}$} & $\begin{array}{l}\text { Sujeta a ataques si no se } \\
\text { construyen reglas de seguridad } \\
\text { adicionales }\end{array}$ & \\
\hline & & & $\begin{array}{l}\text { Difícil integración con acceso a } \\
\text { cliente }\end{array}$ & \\
\hline \multirow{5}{*}{$\begin{array}{l}\text { Aplicación web } \\
\text { residente en servidor }\end{array}$} & PHP & Costos de desarrollo bajos & $\begin{array}{l}\text { Sujeta a múltiples ataques si } \\
\text { no se cuenta con protección } \\
\text { adicional }\end{array}$ & \multirow{5}{*}{8} \\
\hline & \multirow{4}{*}{ asp.net } & $\begin{array}{l}\text { Eliminación de orden impresa } \\
\text { en papel }\end{array}$ & & \\
\hline & & $\begin{array}{l}\text { Capacidad de verificación de } \\
\text { datos en línea }\end{array}$ & & \\
\hline & & Facilidad de implementación & & \\
\hline & & $\begin{array}{l}\text { Facilidad de integración con el } \\
\text { cliente }\end{array}$ & & \\
\hline \multirow{4}{*}{$\begin{array}{l}\text { Aplicación residente } \\
\text { en servidor más } \\
\text { firewall }\end{array}$} & .NET & Costos de desarrollo bajos & Difícil implementación & \multirow{4}{*}{6,5} \\
\hline & $\begin{array}{l}\text { Visual } \\
\text { Basic } \\
\end{array}$ & $\begin{array}{l}\text { Eliminación de orden impresa } \\
\text { en papel }\end{array}$ & $\begin{array}{l}\text { Difícil integración con acceso a } \\
\text { cliente }\end{array}$ & \\
\hline & Java & $\begin{array}{l}\text { Capacidad de verificación de } \\
\text { datos en línea }\end{array}$ & & \\
\hline & Firewalls & $\begin{array}{l}\text { Seguridad de acceso a la } \\
\text { información }\end{array}$ & & \\
\hline
\end{tabular}




\begin{tabular}{|c|c|c|c|c|}
\hline \multirow{4}{*}{$\begin{array}{l}\text { Aplicación web } \\
\text { residente en servidor } \\
\text { más firewall }\end{array}$} & PHP & Costos de desarrollo bajos & $\begin{array}{l}\text { Seguridad adicional solo } \\
\text { proporcionada paraaplicaciones } \\
\text { no relativas al puerto asociado a } \\
\text { ingreso web }\end{array}$ & \multirow{4}{*}{8} \\
\hline & \multirow{3}{*}{ Firewalls } & $\begin{array}{l}\text { Capacidad de verificación de } \\
\text { datos en línea }\end{array}$ & & \\
\hline & & Facilidad de implementación & & \\
\hline & & $\begin{array}{l}\text { Facilidad de integración con el } \\
\text { cliente }\end{array}$ & & \\
\hline \multirow{5}{*}{$\begin{array}{l}\text { Aplicación residente } \\
\text { en servidor más VPN }\end{array}$} & .NET & Costos de desarrollo bajos & \multirow{5}{*}{$\begin{array}{l}\text { Difícil integración con acceso a } \\
\text { cliente }\end{array}$} & \multirow{5}{*}{$\varepsilon$} \\
\hline & $\begin{array}{l}\text { Visual } \\
\text { Basic }\end{array}$ & $\begin{array}{l}\text { Eliminación de orden impresa } \\
\text { en papel }\end{array}$ & & \\
\hline & Java & $\begin{array}{l}\text { Capacidad de verificación de } \\
\text { datos en línea }\end{array}$ & & \\
\hline & \multirow{2}{*}{$\begin{array}{l}\text { VPN (Red } \\
\text { privada } \\
\text { virtual) }\end{array}$} & $\begin{array}{l}\text { Seguridad de acceso a la } \\
\text { información }\end{array}$ & & \\
\hline & & Nivel alto de seguridad & & \\
\hline \multirow{7}{*}{$\begin{array}{l}\text { Aplicación web } \\
\text { residente en servidor } \\
\text { más VPN }\end{array}$} & \multirow{7}{*}{$\begin{array}{l}\text { PHP más } \\
\text { VPN }\end{array}$} & Costo de desarrollo bajo & \multirow{7}{*}{$\begin{array}{l}\text { Posibles vulnerabilidades en } \\
\text { acceso a clientes }\end{array}$} & \multirow{7}{*}{8} \\
\hline & & $\begin{array}{l}\text { Eliminación de orden impresa } \\
\text { en papel }\end{array}$ & & \\
\hline & & $\begin{array}{l}\text { Capacidad de verificación de } \\
\text { datos en línea }\end{array}$ & & \\
\hline & & $\begin{array}{l}\text { Seguridad de acceso a la } \\
\text { información }\end{array}$ & & \\
\hline & & Nivel alto de seguridad & & \\
\hline & & Fácil integración con el cliente & & \\
\hline & & Fácil administración & & \\
\hline
\end{tabular}

\subsection{Componentes}

A partir de la evaluación realizada en la tabla 1, se concluye que para este caso en particular, la solución más apropiada es la aplicación web residente en un servidor que hace uso de canales seguros a través de una VPN [6], para ofrecer servicios de intercambio de información entre los usuarios de la compañía y los clientes, de una manera confiable y oportuna.

El almacenamiento de la información de la compañía se realiza a través de una base de datos desarrollada en el software MySQL. El lenguaje de programación que se usa para el sitio web es PHP [8].

La seguridad de la información se realiza no solamente con la VPN de interconexión remota que usa el modelo cliente-servidor; sino con la aplicación de gestión unificada de amenazas Endian Firewall [9].

\subsection{Modelado}

El diseño del software, involucra la interacción de diversos actores con funciones específicas, como se muestra en la figura 3 [10].

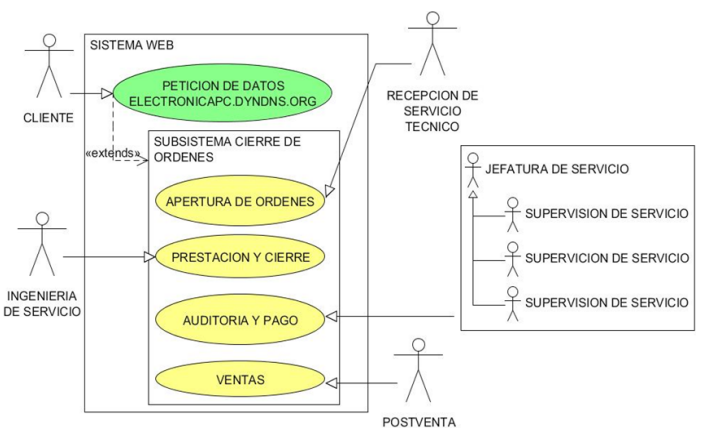

Figura 3. Diagrama de casos de uso del sistema 
El ciclo de vida del software de la figura 4, presenta los procesos principales con base en el modelo cascada [11].

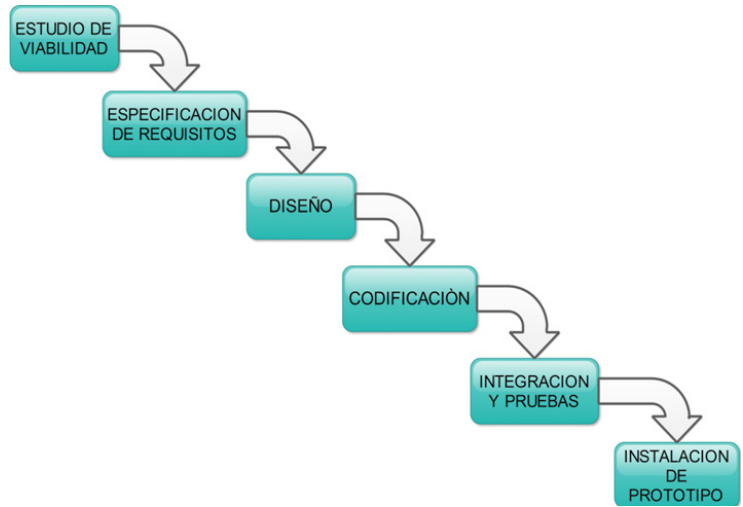

Figura 4. Ciclo de vida del software desarrollado

La base de datos se diseña bajo el modelo entidad-relación, cuyo diagrama se muestra en la figura 5 [8].

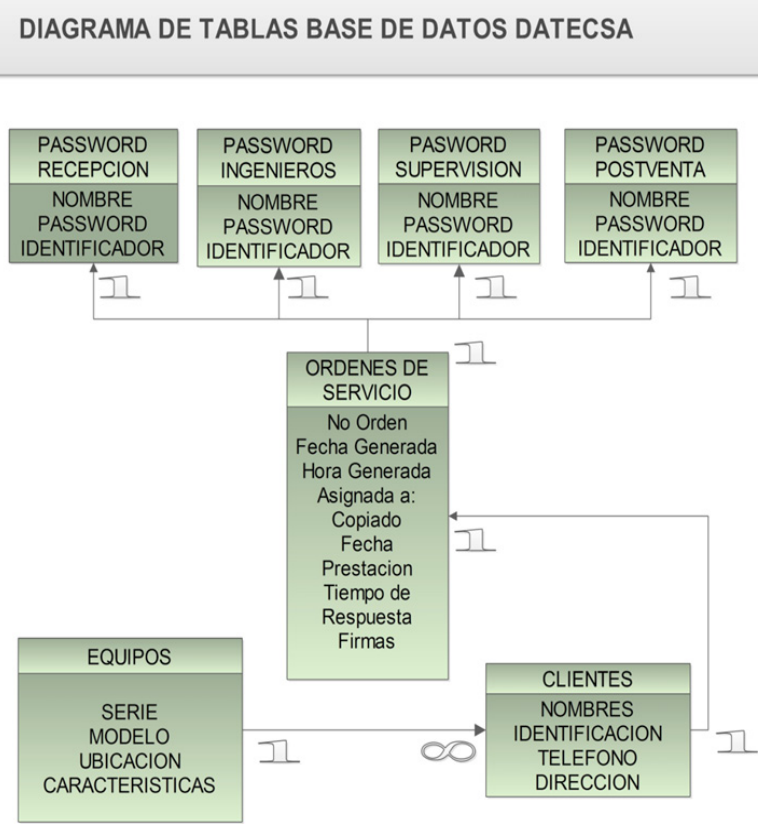

Figura 5. Modelo entidad-relación de la base de datos

\section{DESARROLLO DEL SISTEMA}

Entre los componentes funcionales basados en la interacción hardware/software que están presentes en el sistema, se encuentran los elementos de red y la plataforma de acceso a la información, los cuales se detallan a continuación.

\subsection{Elementos de red}

El diagrama general de direccionamiento y los recursos de la red para lograr una interconexión con los diferentes usuarios, se presentan en la figura 6 [12].

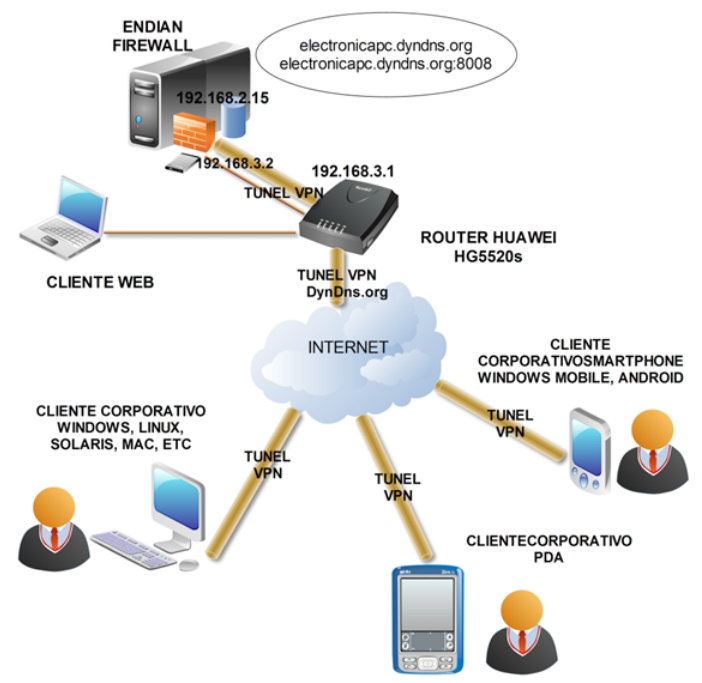

Figura 6. Diagrama general del sistema

\subsection{Plataforma de acceso}

El acceso al sistema de información se realiza vía Web, tanto para los integrantes de la corporación como para los clientes de la compañía, tal como se observa en la figura $7 \mathrm{a}$ y figura $7 \mathrm{~b}$.

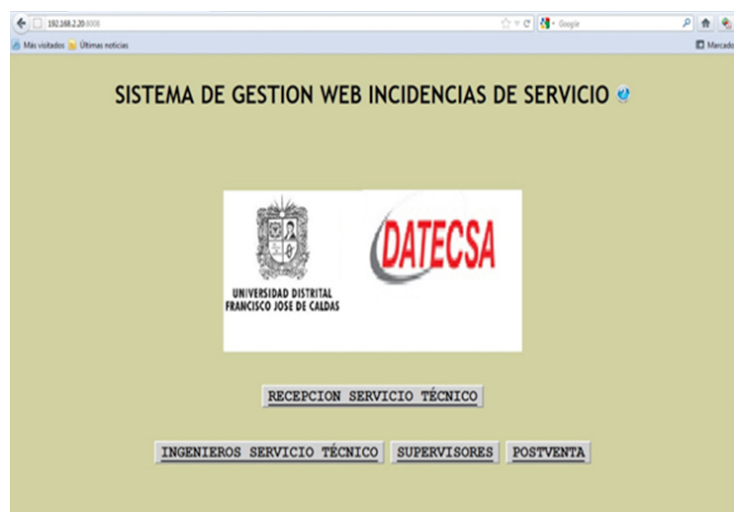

Figura 7a. Plataforma de acceso personal corporativo 


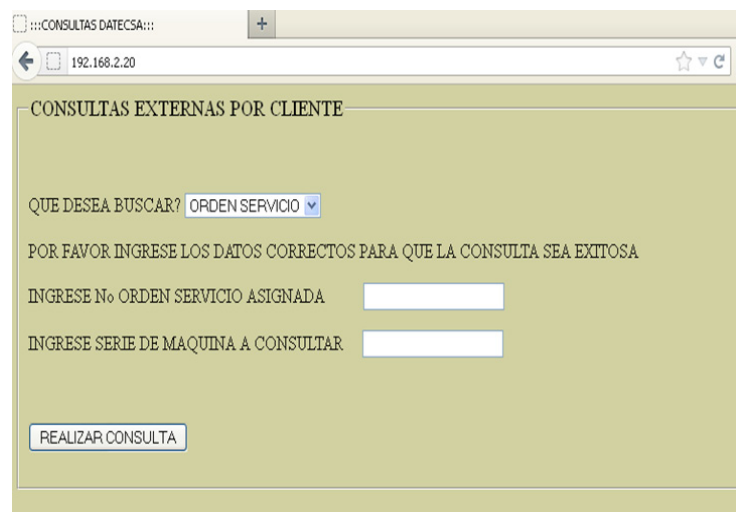

Figura 7b. Plataforma de acceso clientes

\subsection{Componente VPN}

La aplicación para agregarle seguridad a la VPN es Open VPN, implementada sobre la herramienta Endian Firewall, como se ilustra en la figura 8.

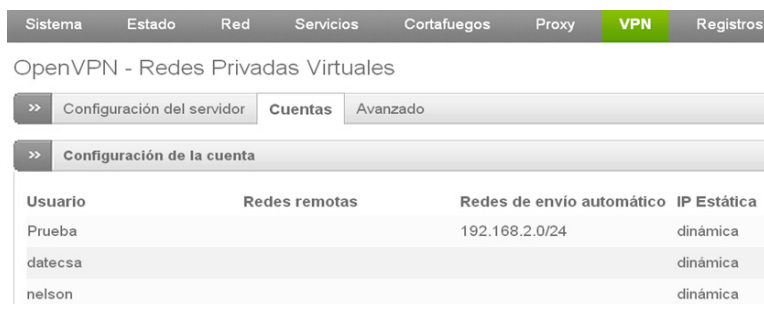

Figura 8. Aplicación Open VPN

Esta aplicación proporciona los protocolos de encriptación SSL/TLS (Secure Sockets Layer/ Transport Layer Security), con el uso de estándares como el denominado AES (Advanced Encryption Standard) con tamaños de clave de 256 bits [12]. El servidor VPN, configurado mediante esta misma herramienta, configura la red para una máquina virtual proporcionando dos interfaces de red virtuales, para los servicios de conexión WAN y LAN. Además, el tráfico de los clientes es direccionado a través de este servidor.

\section{RESULTADOS}

En esta sección se presentan los resultados de las diferentes pruebas de seguridad realizadas al sistema implementado.

\subsection{Análisis de vulnerabilidad}

Inicialmente, la seguridad de la infraestructura se evalúa con una herramienta para la detección de vulnerabilidades, enfocada a encontrar fallas en los módulos de gestión y cliente, de la plataforma desarrollada. En la figura 9, se muestra que luego del análisis realizado no se encontraron vulnerabilidades conocidas.

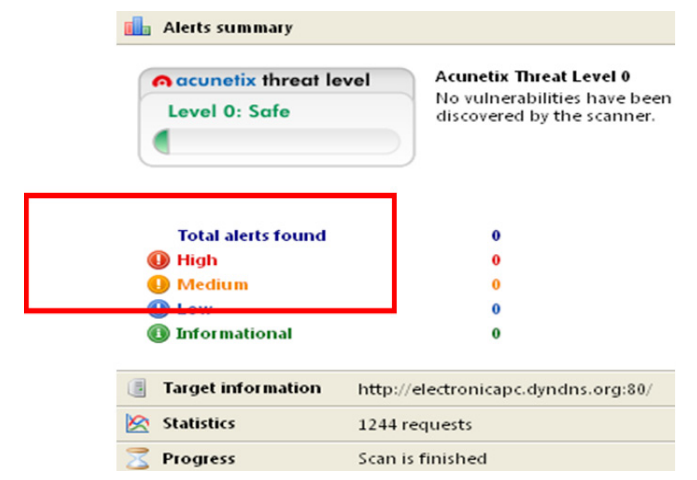

Figura 9. Resultado de vulnerabilidades

\subsection{Rastreo de puertos}

Se ejecutó una prueba de seguridad con la herramienta Nmap, con el fin de revisar la información acerca de los puertos abiertos, lo cual puede conllevar al acceso de intrusos en el sistema. En la figura 10, se advierte que no se puede acceder a los puertos del sistema, lo que demuestra la robustez en la seguridad del sistema.

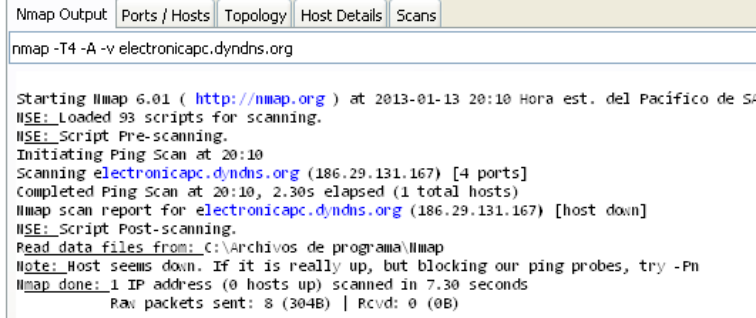

Figura 10. Rastreo de puertos

\subsection{Rastreo de tráfico}

A través de la herramienta Wireshark, se verifica la ininteligibilidad de la información cuan- 
do es accedida por un intruso, el tráfico usado para este caso corresponde a una comunicación cliente-servidor, tal como se presenta en la figura 11.

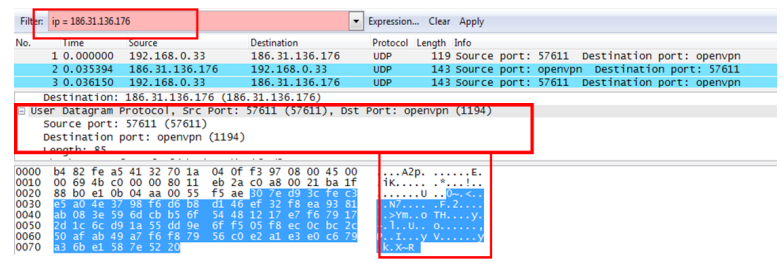

Figura 11. Rastreo de tráfico filtrado por dirección IP.

\subsection{Conexión de clientes}

Finalmente, se verifica las características de conexión de un cliente a la base de datos ubicada en el servidor. En la figura 12 se observan los resultados de la conexión.

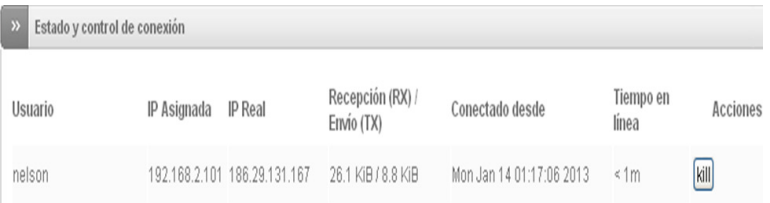

Figura 12. Características de conexión de un cliente a través de la VPN, al servidor del sistema

\section{CONCLUSIONES}

El estudio realizado a la empresa Datecsa S.A, para la implementación de su sistema de infor-

Referencias Bibliográficas

[1] M. Korpela, A. Mursu, H.A. Soriyan; Information systems development as an activity, Computer Supported Cooperative Work, vol. 11, no.1-2, pp 111 - 128, 2002.

[2] J. Muñoz; Information systems development methodologies for Datadriven Decision Support Systems, Thesis to obtain the degree of MSc in Managementand Information systems, University of Manchester, 2010.

[3] I.M. Yassin, A. Zabidi, M.A. Johari, F. Hijaz, A.H. YussufIzzudin, N.M. Tahir, M.K. Hamzah, A. Majeed; Entity- mación, entrego como mejor opción el desarrollo de una plataforma de acceso web, con protección de la información a través de una VPN.

El desarrollo satisfactorio del sistema de información incluyó la utilización de herramientas como MySQL, HTML y PHP, para el acceso y el almacenamiento de la información, con las que se satisficieron las necesidades del proyecto.

En la fase de análisis se identificaron todos los actores que intervienen en el sistema, permitiendo concebir los requerimientos y características que se necesitaban para el desarrollo de una herramienta robusta.

Se estableció un mecanismo de seguridad para la administración y acceso de la información dentro del sistema, controlando las modificaciones y/o visualización de la información, teniendo en cuenta los privilegios del usuario.

El sistema se desarrolló con herramientas multiplataforma, con el fin de que no existan inconvenientes en el acceso para los usuariosque usansistemas UNIX/Linux ó Windows.

\section{AGRADECIMIENTOS}

A la compañía nacional de prestación de servicios integrales Datecsa S.A.

Relationship analysis for development of Malaysian University Research Assessment Instrument (MyRA) information system, IEEE International Conference on System Engineering and Technology (ICSET), pp 219-224, 2011.

[4] L. Rocco; Análisis, diseño e implementación de una solución para administración de operaciones en terreno usando dispositivos móviles y mensajería SMS, Tesis para optar al título de Ingeniero Civil en Informática, Universidad Austral de Chile, 2005. 
[5] Manual de PHP. [En línea], Consultado en Noviembre 18 de 2012, disponible en: http://php.net/manual/es/index.php.

[6] J. Alonso; Redes privadas virtuales, Editorial RA-MA, Madrid, pp.15-20, 2009.

[7] W. Stallings W; Network security essentials: Applications and Standards, cuarta edición, Editorial Pearson Education, New Jersey, pp 75-90, 2011.

[8] J. Meloni; Sams teach yourself PHP, MySQL and Apache all in one, quinta edición, Editorial Sams Publishing, Indianapolis, pp 18-22, 2012.
[9] Endian UTM Appliance 2.5 reference manual. [En línea], Consultado en Septiembre 10 de 2012, disponible en: http://docs.endian.com/

[10] G. Booch J. Rumbaugh, I. Jacobson; El lenguaje unificado de modelado, primera edición, Editorial Addison Wesley, Madrid, pp 50-58, 2000.

[11] R. Pressman; Ingeniería del software, sexta edición, Editorial McGraw Hill, México D.F, pp 68-79, 2006.

[12] V. Bollapragada M. Khalid, S. Wainner; IPSec VPN design, segunda edición, Editorial Cisco Press, Indianapolis, pp 78-87, 2006. 